\title{
Synthesis of electromagnetic metasurfaces: principles and illustrations
}

\author{
Karim Achouri ${ }^{1, *}$, Bakthiar Ali Khan ${ }^{1}$, Shulabh Gupta ${ }^{1}$, Guillaume Lavigne ${ }^{1}$, \\ Mohamed Ahmed Salem ${ }^{1}$, and Christophe Caloz ${ }^{1,2}$ \\ 1 Dept. of Electrical Engineering, Polytechnique Montréal, Montréal, QC H2T 1J3, Canada \\ 2 Electrical and Computer Engineering Dept. King Abdulaziz University, P.O. Box 80204, Jeddah 21589, Saudi Arabia
}

Received 1 September 2015 / Accepted 19 November 2015

\begin{abstract}
The paper presents partial overview of the mathematical synthesis and the physical realization of metasurfaces, and related illustrative examples. The synthesis consists in determining the exact tensorial surface susceptibility functions of the metasurface, based on generalized sheet transition conditions, while the realization deals with both metallic and dielectric scattering particle structures. The examples demonstrate the capabilities of the synthesis and realization techniques, thereby showing the plethora of possible metasurface field transmission and subsequent applications. The first example is the design of two diffraction engineering birefringent metasurfaces performing polarization beam splitting and orbital angular momentum multiplexing, respectively. Next, we discuss the concept of the electromagnetic remotely controlled metasurface spatial processor, which is an electromagnetic linear switch based on destructive interferences. Then, we introduce a non-reciprocal non-gyrotropic metasurface using a pick-up circuit radiator (PCR) architecture. Finally, the implementation of all-dielectric metasurfaces for frequency dispersion engineering is discussed.
\end{abstract}

Key words: Metasurface synthesis, Birefringence, Refraction, Orbital angular momentum, Frequency dispersion engineering, Metamaterial switch, Non-reciprocal metadevice.

\section{Introduction}

Metasurfaces, which are the two-dimensional counterparts of volume metamaterials [1-3], have attracted much attention over the past years. Due to their low profile, small losses and rich electromagnetic field manipulation capabilities, they are excellent spatial processors able to manipulate electromagnetic waves with ever more complex possible applications, such as generalized refraction, polarization transformation, signal multiplexing, and non-reciprocal field control.

Metasurfaces are usually made of uniform or non-uniform arrangements of specifically engineered sub-wavelength scattering particles to produce a desired scattered field. Many such metasurfaces have been reported. However, these metasurface were most often designed using heavy optimization techniques. Fortunately, a few efficient synthesis techniques have been recently introduced, offering powerful and versatile tools for metasurface design [4-8].

In this paper, we briefly recall the synthesis technique which is detailed in reference [4]. Next, we illustrate the capabilities and the advantages of this synthesis technique.
The various metasurfaces addressed here will be described and analysed more thoroughly in other future publications.

\section{Metasurface design}

\subsection{Susceptibility synthesis}

Let us consider that a metasurface lying in the $x-y$ plane at $z=0$. Given its inherent sub-wavelength thickness, a metasurface may be modeled as a zero-thickness spatial discontinuity. Rigorous boundary conditions, pertaining to such a discontinuity, were first derived by Idemen [9] and later applied to metasurfaces by Kuester et al. [10]. Conventionally called the Generalized Sheet Transitions Conditions (GSTCs), they read

$$
\begin{gathered}
\hat{z} \times \Delta \boldsymbol{H}=j \omega \boldsymbol{P}_{\|}-\hat{z} \times \nabla_{\|} M_{z}, \\
\Delta \boldsymbol{E} \times \hat{z}=j \omega \mu \boldsymbol{M}_{\|}-\nabla_{\|}\left(\frac{P_{z}}{\varepsilon}\right) \times \hat{z}, \\
\hat{z} \cdot \Delta \boldsymbol{D}=-\nabla \cdot \boldsymbol{P}_{\|},
\end{gathered}
$$

*e-mail: karim.achouri@polymtl.ca

This is an Open Access article distributed under the terms of the Creative Commons Attribution License (http://creativecommons.org/licenses/by/4.0), which permits unrestricted use, distribution, and reproduction in any medium, provided the original work is properly cited. 


$$
\hat{z} \cdot \Delta \boldsymbol{B}=-\mu \nabla \cdot \boldsymbol{M}_{\|}
$$

where the time dependence $e^{j \omega t}$ is dropped by convenience. The permittivity $\varepsilon$ and the permeability $\mu$ correspond to the medium surrounding the metasurface. The sign $\Delta$ corresponds to the difference of the electromagnetic fields $(\boldsymbol{E}, \boldsymbol{H}$, $\boldsymbol{D}$ and $\boldsymbol{B})$ between both sides of the metasurface, $\boldsymbol{P}$ and $\boldsymbol{M}$ are the electric and magnetic polarization densities, respectively. The subscript $\|$ refers to the vectorial components that are in the plane of the metasurface. The synthesis technique [4] is based on the relations (1a)-(1d) and determines the susceptibilities required to perform the specified wave transformations.

Relations (1a)-(1d) form a set of coupled partial differential equations. To simplify the analysis, we consider that the metasurface is composed of only surface polarization densities, which reduces relations (1a) and (1b) to a set of coupled linear equations that can be solved for closed-form expressions of the metasurface susceptibilities. Upon substitution of the general definitions of $\boldsymbol{P}$ and $\boldsymbol{M}$, in terms of the bi-anisotropic susceptibility tensors $\overline{\bar{\chi}}_{\text {ee }}, \overline{\bar{\chi}}_{\text {mm }}, \overline{\bar{\chi}}_{\text {em }}$ and $\overline{\bar{\chi}}_{\text {me }}$ [11], (1a)-(1d) reduces to

$$
\begin{gathered}
\hat{z} \times \Delta \boldsymbol{H}=j \omega \varepsilon \overline{\bar{\chi}}_{\mathrm{ee}} \boldsymbol{E}_{\mathrm{av}}+j k \overline{\bar{\chi}}_{\mathrm{em}} \boldsymbol{H}_{\mathrm{av}}, \\
\Delta \boldsymbol{E} \times \hat{z}=j \omega \mu \overline{\bar{\chi}}_{\mathrm{mm}} \boldsymbol{H}_{\mathrm{av}}+j k \overline{\bar{\chi}}_{\mathrm{me}} \boldsymbol{E}_{\mathrm{av}},
\end{gathered}
$$

where the polarizations densities $\boldsymbol{P}$ and $\boldsymbol{M}$ are expressed in terms of the arithmetic average of the fields (as denoted by the subscript "av") instead of the acting or total fields at the position of each scattering particles [10].

The mathematical synthesis of the metasurface essentially consists in inserting the electromagnetic fields of the specified transformations into (2a) and (2b) and solving for the susceptibilities. Depending on the desired transformation, the system of equations (2a) and (2b) may be under-determined, in which case some of the tensor elements must be set to zero for a definite solution [4]. Once the susceptibilities of the metasurface are determined, the metasurface can be physically realized, which is the topic of the next section.

\subsection{Scattering particle synthesis and implementation}

The physical realization of metasurfaces is, as of today, not a trivial routine. Here, two possible approaches are briefly discussed, while many more exist. In both approaches, the susceptibility functions, obtained by the aforementioned synthesis procedure, are spatially discretized in the $x-y$ plane where each discrete point corresponds to a unit cell to be implemented. The unit cells are simulated (assuming periodic boundary conditions), one by one or group by group when the structure is fully or partly non-uniform, using commercial softwares that compute their scattering parameters. The required physical parameters for the scattering particles are obtained by mapping the scattering parameters into the susceptibility function [4]. Finally, an enhanced design is achieved by tuning the parameters of the scattering particles via parametric analysis or standard optimization techniques.

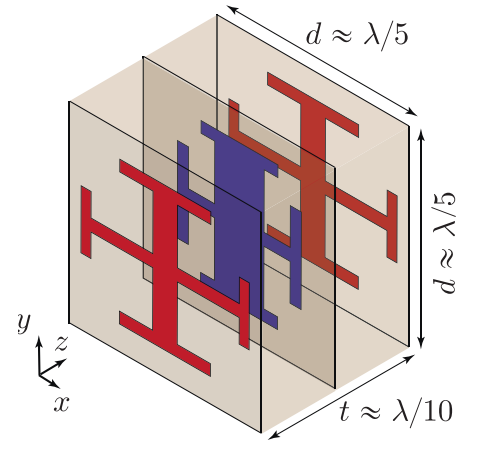

Figure 1. Generic unit cell with three metallic (PEC) Jerusalem crosses separated by dielectric slabs, the outer layers are identical.

\subsubsection{Metallic scatterers}

Metasurfaces consisting of metallic scatterers, typically on dielectric substrates, have been the most commonly reported types of metasurfaces, as well as their predecessors, the frequency selective surfaces (FSS) [12]. In all cases, using more than one layer is an effective way to increase the available number of degrees of freedom, and hence achieving enhanced properties, including higher bandwidth and larger phase coverage range of the structure's unit cell. It was recently shown that three cascaded layers, where the two outer layers are identical, represents the minimum configuration to achieve full transmission and a $2 \pi$ phase coverage $[13,14]$. With three layers, the overall thickness of the metasurface generally remains subwavelength (usually in the order of $t \approx \lambda / 10$ ) with negligible loss increase. Further increasing the number of layers may naturally be an approach for even broader bandwidth, at the expense of loss and weight.

A typical shape for the scattering particles forming the metallic layers is the Jerusalem cross, as shown in Figure 1. The Jerusalem cross has the advantage of featuring fairly well decoupled responses for $x$ and $y$ polarizations, consequently simplifying the implementation. In the structure of Figure 1, relatively strong capacitive coupling in the transverse $(x-y)$ plane offers the benefit of lowering the resonance frequencies $[12,15]$ or, alternatively, of reducing the free-space electrical size of the unit cell $(d \approx \lambda / 5)$, while introducing more complexity in terms of coupling.

The realization of the unit cells is greatly simplified if the longitudinal evanescent mode coupling between the three metallic layers can be minimized. This occurs when the dielectric spacer relative permittivity is decreased or/and its thickness is increased. In that case, each layer can be designed separately and the overall response of the multi-layer unit cell can then be found using simple transmission matrix approaches [14].

\subsubsection{Dielectric scatterers}

The second implementation method is based on alldielectric scattering particles. It has been known for a long time that dielectric resonators exhibit both electric and magnetic resonances $[16,17]$. More recently, dielectric resonators have been used to realize all-dielectric metasurfaces $[18,19]$. A typical unit cell is shown in Figure 2a where the particles 


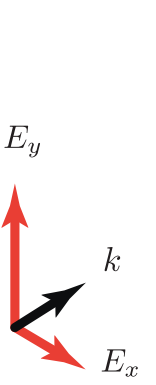

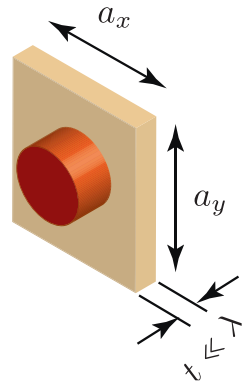

(a)

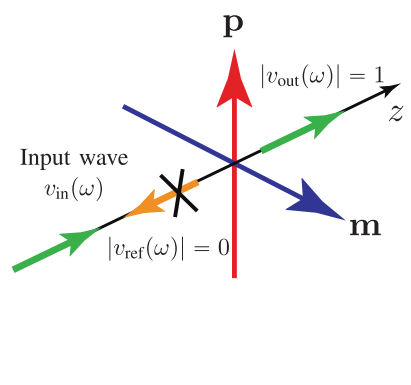

(b)
Figure 2. (a) Representation of an all-dielectric all-pass metasurface unit cell consisting of a dielectric resonator $\left(\varepsilon_{r, 1}\right)$ embedded in a host layer of permittivity $\varepsilon_{r, 2}$. (b) Operation principle for full transmission (zero reflection).

are dielectric cylinders of circular cross section with permittivity $\varepsilon_{r, 1}$ embedded in a substrate with permittivity $\varepsilon_{r, 2}$. Other types of particle shapes are naturally also possible. It is possible, by tuning the physical dimensions of the resonator as well as the permittivities ratio $\varepsilon_{r, 1} / \varepsilon_{r, 2}$, to merge the electric and magnetic resonances. In this scenario, if the two resonances have the same strength and are associated with orthogonal dipole moments in the transverse plane of the metasurface, as shown in Figure $2 b$, reflection may be totally suppressed. This is due to perfect destructive interference of the waves scattered by the electric and magnetic scattering particles in the incident side of the metasurface, and their constructive interference at the transmission side of it [17]. In this case, the transmission is theoretically $100 \%$ and flat over a wide bandwidth. Moreover, the transmission phase covers a full $2 \pi$ range around the resonance frequency $\omega$. This powerful concept may therefore apply to all-pass metasurfaces with controllable phase over large bandwidth. It must be noted that, in general, the electric and magnetic resonances have different dispersion [20] which leads to a transmission amplitude that is not perfectly flat over the bandwidth of interest. However, such small variations in transmission may be in general neglected since the transmission coefficient $S_{21}$ does not drop below $-2 \mathrm{~dB}$ (assuming no dielectric loss), as will be shown in Section 3.4.

Structurally symmetric shapes like cylinders or squares present the same behavior for $x$ - and $y$-polarized waves. However, using asymmetric shapes, such as ellipses and rectangles, allow for a complete and independent control of the two orthogonal polarizations, as recently demonstrated in reference [19]. As an additional advantage, dielectric unit cells have a greatly reduced number of physical parameters to adjust compared to the three layer Jerusalem crosses of Figure 1, effectively simplifying the optimization procedure to achieve the specified response.

\section{Illustrative examples}

\subsection{Birefringent metasurface}

The first example we present is a birefringent metasurface. Birefringence is the property, available in certain crystal, according to which different refractive indices are presented to different wave polarizations. This property requires anisotropy [21]. A birefringent crystal has, in the plane transverse to propagation, a fast axis and slow axis corresponding to low and high refractive indices, respectively. Thus, a wave polarized along the direction of the fast axis undergoes a smaller phase shift than a wave polarized along the slow axis. Based on this principle, several interesting devices can be realized. For example, a half-wave plate, which induces a $\pi$ phase shift between the two orthogonal polarizations so as to change the polarization angle of linearly polarized waves (rotation of polarization without chirality) or change the handedness of circularly polarized waves. Similarly, a quarter-wave plate induces a $\pi / 2$ phase shift between the two polarizations, which allows transforming a linearly polarized wave into a circularly polarized wave or vice versa.

Birefringent metasurfaces can be mathematically described using the synthesis technique of Section 2.1. Since bi-anisotropy (or chirality) are not required to achieve birefringence, (2a) and (2a) reduces to

$$
\begin{gathered}
\left(\begin{array}{c}
-\Delta H_{y} \\
\Delta H_{x}
\end{array}\right)=j \omega \varepsilon\left(\begin{array}{cc}
\chi_{\mathrm{ee}}^{x x} & 0 \\
0 & \chi_{\mathrm{ee}}^{y y}
\end{array}\right)\left(\begin{array}{c}
E_{x, \mathrm{av}} \\
E_{y, \mathrm{av}}
\end{array}\right), \\
\left(\begin{array}{c}
\Delta E_{y} \\
-\Delta E_{x}
\end{array}\right)=j \omega \mu\left(\begin{array}{cc}
\chi_{\mathrm{mm}}^{x x} & 0 \\
0 & \chi_{\mathrm{mm}}^{y y}
\end{array}\right)\left(\begin{array}{c}
H_{x, \mathrm{av}} \\
H_{y, \mathrm{av}}
\end{array}\right) .
\end{gathered}
$$

Following the procedure described in reference [4], the system is straightforwardly solved and yields two sets of orthogonal susceptibilities, given for $x$-polarized waves by

$$
\begin{gathered}
\chi_{\mathrm{ee}}^{x x}=\frac{-\Delta H_{y}}{j \omega \varepsilon E_{x, \mathrm{av}}}, \\
\chi_{\mathrm{mm}}^{y y}=\frac{-\Delta E_{x}}{j \omega \mu H_{y, \mathrm{av}}},
\end{gathered}
$$

and for $y$-polarized waves by

$$
\begin{gathered}
\chi_{\mathrm{ee}}^{y y}=\frac{\Delta H_{x}}{j \omega \varepsilon E_{y, \mathrm{av}}}, \\
\chi_{\mathrm{mm}}^{x x}=\frac{\Delta E_{y}}{j \omega \mu H_{x, \mathrm{av}}} .
\end{gathered}
$$

We shall next consider two specific types of birefringences, generalized refraction and orbital angular momentum birefringences, that are both achieved by using proper non-uniform susceptibilities in (4a)-(5b).

\subsubsection{Generalized refraction birefringence}

The concept of generalized refraction birefringence is proposed here as a direct application of the general synthesis technique described in reference [4] and simplified for the case of birefringence in (4a)-(5b). Generalized refraction birefringence consists in independently and simultaneously controlling the reflection and the transmission coefficients of $x$-polarized 


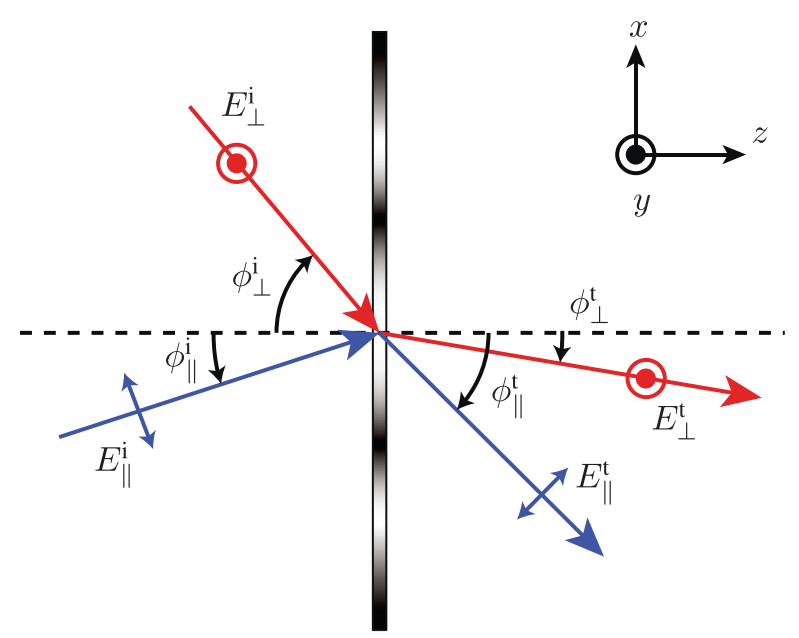

Figure 3. Example of a birefringent "generalized refraction". Two orthogonally polarized plane waves incident on the metasurface get refracted at different and independent angles.

and $y$-polarized plane waves incident on the metasurface. The principle is illustrated in Figure 3, where the reflected waves are specified to be zero.

As an example, we present the implementation of a polarization beam splitter (or, reciprocally, a polarization combiner). The corresponding metasurface, that is shown in Figure 4, is first described in terms of its surface susceptibilities, by inserting the specified incident, reflected and transmitted electric and magnetic fields into (4a)-(5b). Here, the reflected waves are specified to be zero and the transmitted waves are specified to be fully refracted at $\phi_{\perp}=+60^{\circ}$ and $\phi_{/ /}=-60^{\circ}$, respectively, in the diagonal of the square metasurface. The computed susceptibilities, which are periodic in the $x-y$ plane, are then discretized into eight fundamental unit cells of sides $d \approx \lambda / 5$ and thickness $t \approx \lambda / 10$. Following the procedure described in Section 2.2.1, each unit cell is designed separately to realize the required equivalent surface susceptibilities. The fabricated metasurface, shown in Figure 4b, consists in $24 \times 24$ unit cells. From periodicity, the unit cells can be grouped into periodic supercells composed of $8 \times 8$ unit cells.

Because the metasurface in Figure $4 \mathrm{~b}$ is electrically too large to be simulated, a simplified version of the metasurface is considered for the full-wave simulations. Only the first row of the $8 \times 8$ supercell is selected. Numerical simulations (CST) are performed for $x$ and $y$ polarizations. The results are shown in Figures 5a and 5b, respectively. It has to be noted that, because the periodicity is now only in the $x$ direction, instead of being in the diagonal of the supercell, the refraction angle is reduced. This can be understood by considering that the projected phase of the refracted wave on the metasurface undergoes one full cycle along the $x$ direction but two full cycles along the diagonal of the supercell.

As may be seen in Figure 5a, the simulation is in good agreement with the expected result. However, the simulation in Figure $5 \mathrm{~b}$ shows a refracted wave perturbed by the presence of a zeroth diffraction order contribution. The reason for the mediocre results of Figure $5 \mathrm{~b}$ is the altered coupling between

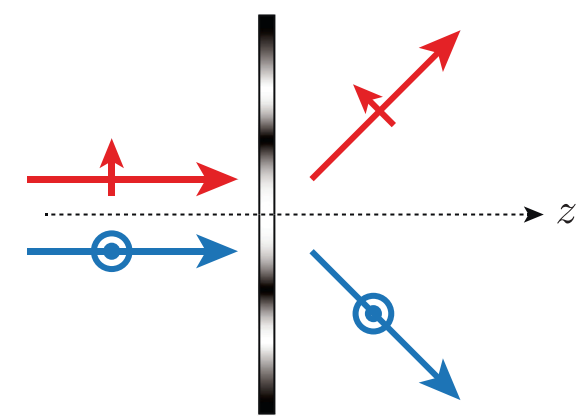

(a)

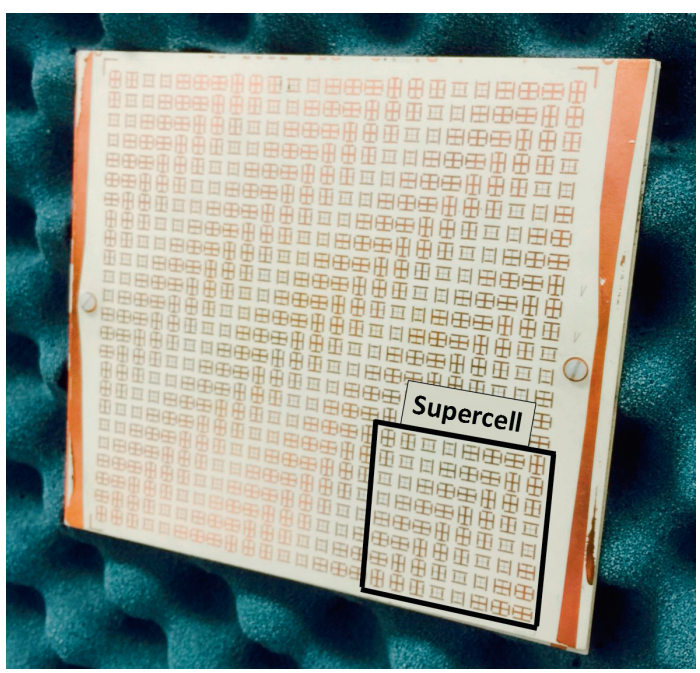

(b)

Figure 4. (a) Representation of the polarization beam splitter/ combiner. (b) Fabricated structure with $24 \times 24$ unit cells. The two polarizations are refracted in the diagonal direction where a macroscopic stripe-like pattern is apparent.

the unit cells due to truncation, which yields the observed zeroth order transmission.

\subsubsection{Orbital angular momentum birefringence}

The second example for a birefringent metasurface is an orbital angular momentum (OAM), or vortex wave, multiplexing metasurface [22]. The generation of OAM waves using metasurfaces has been presented in several recent reports [23-25]. Bessel waves are a typical choice for vortex waves. However, Bessel waves are either radially or azimuthally polarized, demanding for either inhomogeneous rotation of polarization if the incident plane wave is linearly polarized, or that the incident plane wave be circularly polarized. To avoid this constraint, Hypergeometric-Gaussian (HyG) waves are considered instead. The electric field of a HyG wave is given by [26]

$$
\begin{aligned}
E(\rho, \phi, z)= & \frac{\Gamma\left(1+|m|+\frac{p}{2}\right)}{\Gamma(|m|+1)} \frac{i^{|m|+1} \zeta^{|m| / 2} \xi^{p / 2}}{[\xi+i]^{1+|m| / 2+p / 2}} e^{i m \phi-i \zeta} \\
& \times{ }_{1} F_{1}\left(-\frac{p}{2},|m|+1 ; \frac{\zeta[\xi+i]}{\xi[\xi-i]}\right),
\end{aligned}
$$




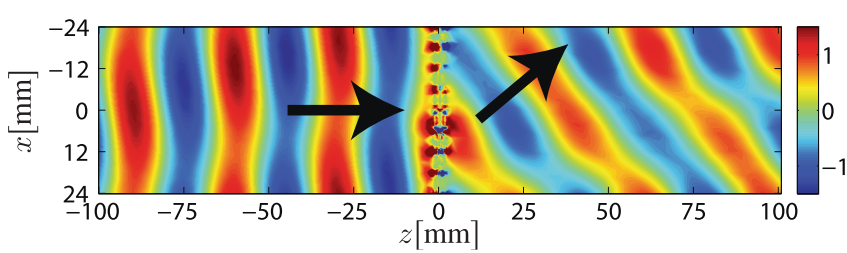

(a)

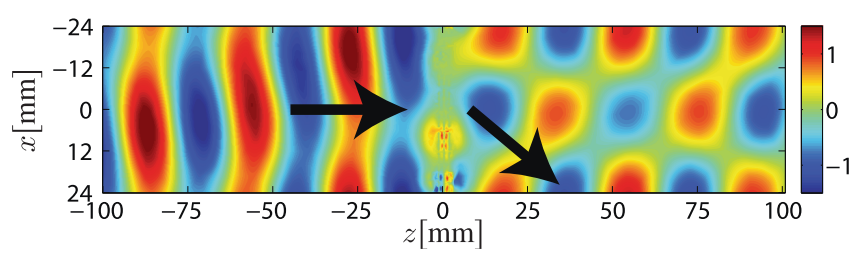

(b)

Figure 5. Full-wave simulations, assuming periodic boundary conditions, of the first row of the supercell shown in Figure 4b. (a) An $x$-polarized normally incident plane wave is impinging from left to right on the metasurface that refracts it upward. (b) A $y$-polarized normally incident plane wave is impinging from left to right on the metasurface that refracts it downward.

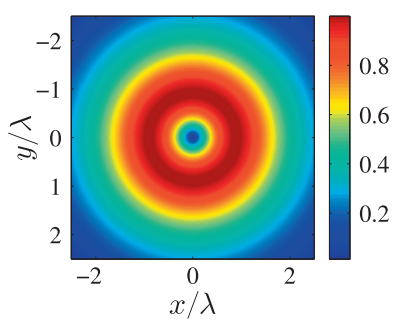

(a)

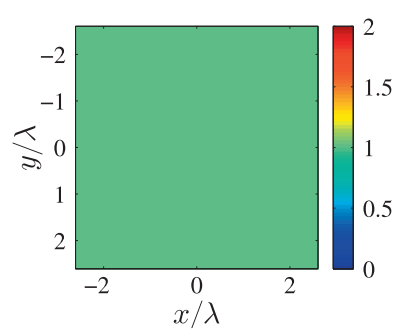

(c)

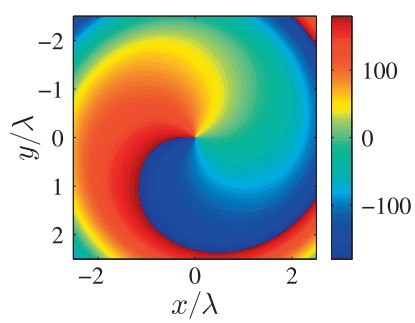

(b)

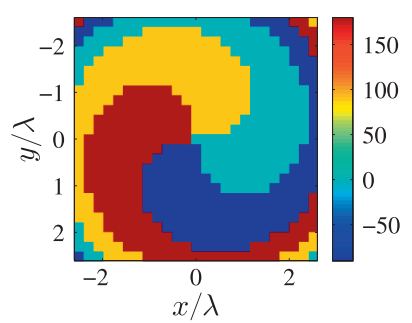

(d)
Figure 6. Magnitude (a) and phase (b) of the HypergeometricGaussian (HyG) wave with parameters $p=1, m=-1, w_{0}=\lambda$, $\xi=1$. Simplified version of the specified fields with flat magnitude (c) and four phase samples (d).

where ${ }_{1} F_{1}(a, b ; x)$ is the confluent hypergeometric function, $\Gamma(x)$ is the gamma function, $m$ is the OAM order, $p \geq-|m|$ is a real parameter, and where $\zeta=\rho^{2} /\left(w_{0}^{2}[\xi+i]\right), \xi=z / z_{r} w_{0}$, with $w_{0}$ being the beam waist and $z_{r}$ the Rayleigh range given by $z_{r}=\pi w_{0}^{2} / \lambda$.

Compared to Bessel waves, HyG waves have the advantage of being linearly polarized, thus allowing direct transformation (i.e. no rotation of polarization) from a linearly polarized plane wave, as well as the multiplexing of orthogonal plane waves into orthogonal vortex HyG of different order $m$.

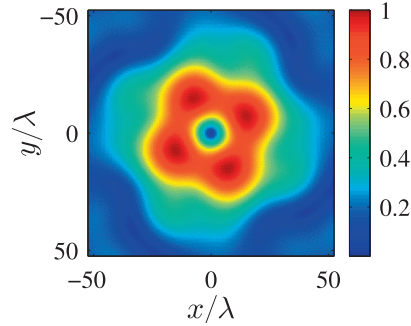

(a)

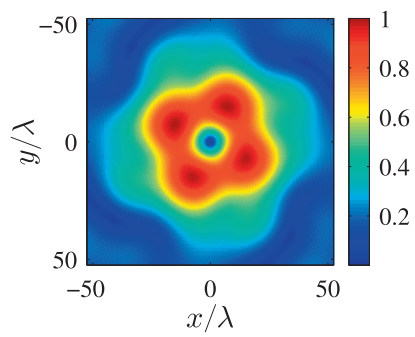

(c)

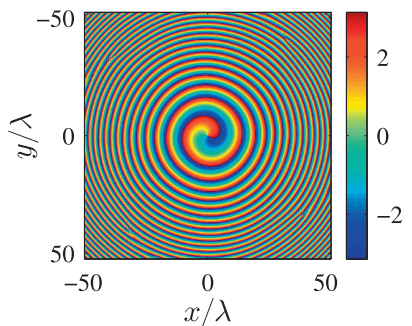

(b)

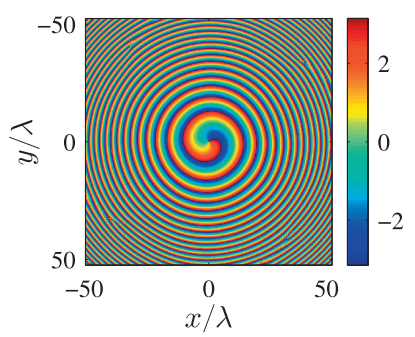

(d)
Figure 7. Fourier propagated (a) magnitude and (b) phase, at a distance of $z=100 \lambda$, generated by the simplified fields of Figures $6 \mathrm{c}$ and $6 \mathrm{~d}$. Fourier propagated (c) magnitude and (d) phase, at a distance of $z=100 \lambda$, for the HyG wave of order $m=+1$. The four stronger regions in (a) and (c) are due to the square shape of the aperture formed by the metasurface (size $5 \lambda \times 5 \lambda$ ). Using a circular metasurface would result in a better approximation of the ideal doughnut shaped magnitude of Figure 6a.

Consider the following transformation, where two normally incident plane waves, polarized along $x$ and $y$, respectively, are transformed into HyG waves of order $m=+1$ and $m=-1$, respectively. Figure 6 shows the typical amplitude and phase distributions of a vortex $\mathrm{HyG}$ wave of order $m=-1$. Because of its complex structure, a complete discretization of the HyG wave would result in a very large number of scattering particles. In order to simplify the problem, the $\mathrm{HyG}$ wave phase is coarsely sampled with only four phase samples, as shown in Figure 6d. The four phase samples are sufficient, according to the Nyquist criterion, to describe the full phase evolution of the wave. As for the magnitude of the HyG wave, it is transformed into a flat uniform plane of magnitude $|E|=1$, as shown in Figure 6c. Although the implemented magnitude is flat, the radiated field magnitude variations follow naturally from self-interference.

It can be easily shown that, even with such a drastic simplification, the fields scattered by the metasurface do exhibit the smooth shape of the continuous fields of Figures $6 \mathrm{a}$ and $6 \mathrm{~b}$. This can be verified by Fourier propagating the fields of Figures $6 \mathrm{c}$ and $6 \mathrm{~d}$, as shown in Figure 7. Similar considerations apply to the HyG wave of order $m=+1$ whose phase spirals in the opposite direction than the phase of the HyG wave of order $m=-1$.

The final metasurface is implemented by combining both $m=+1$ and $m=-1$ HyG waves, which results in a total number of 16 different unit cells distributed over the area of the metasurface. The final fabricated structure is shown in Figure 8 . 


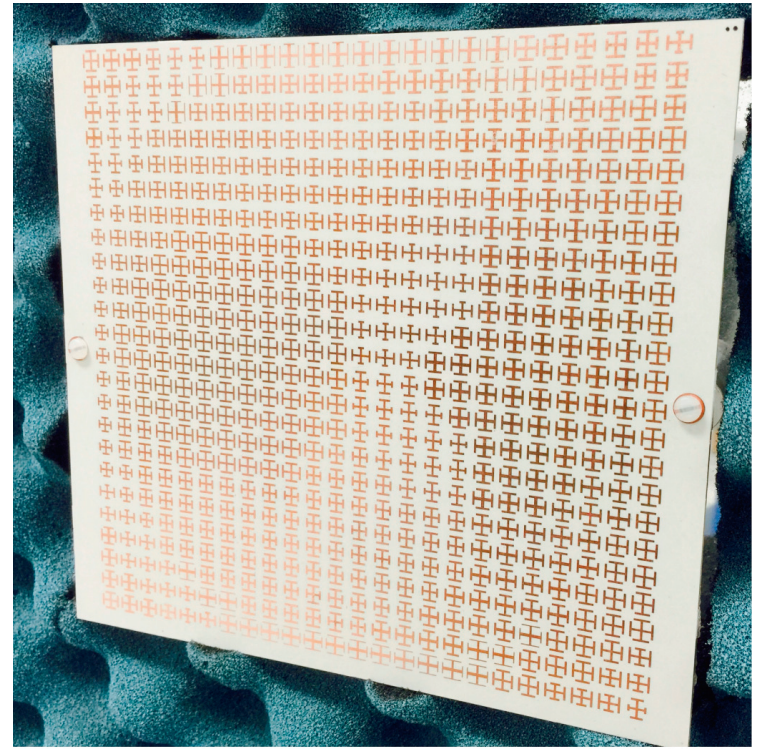

Figure 8. Fabricated OAM multiplexing metasurface.

\subsection{Electromagnetic remotely controlled spatial processor}

In this section, we propose to synthesize and implement a metasurface spatial processor whose transmitted wave (or reflected wave) can be remotely modified by the presence of a control incident wave. The metasurface has two main operation states, with or without control wave. In the most general case, all waves can be arbitrarily specified and the control wave can therefore dramatically modify the initial transmitted wave. Moreover, changing the amplitude, phase or polarization of the control wave separately can be used as additional degrees of freedom to tune the transmitted wave. The metasurface itself is not necessarily tunable, in contrast to certain structures with tunable scattering particles [27-30]. Likewise, the electromagnetic properties of the metasurface are not modified by the presence of the control wave in contrast to, for instance $[31,32]$.

Consider for instance the metasurface spatial processor illustrated in Figure 9. In this example, the reflected waves are specified to be zero. When no control wave is present, the metasurface transforms an incident wave (or signal) into a transmitted wave refracted at a $45^{\circ}$ angle with respect to the normal of the metasurface, as shown in Figure 9a. When the control wave is impinging on the metasurface, as in Figure 9b, the transmitted wave refraction angle becomes $-45^{\circ}$.

We now present a simpler operation for the metasurface spatial processor concept: switching. Consider two plane waves, one $x$-polarized (the signal wave) and one $y$-polarized (the control wave), both normally incident on the metasurface. The signal wave is normally transmitted by the metasurface, whereas the control wave is also normally transmitted but its polarization is rotated to match the polarization of the transmitted signal wave. The control is achieved by destructive interference between the signal and the control waves, as shown in Figure 10a. In the configuration of Figure 10a, the signal wave and the control wave have the same point of incidence and

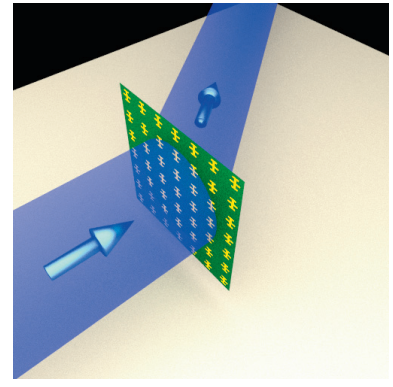

(a)

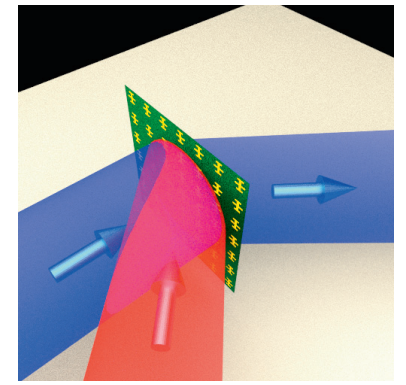

(b)
Figure 9. Electromagnetic remotely controlled metasurface concept. The metasurface is represented as an array of Jerusalem crosses. In this example, zero reflection is specified. (a) An incident wave (or signal) is transformed into a transmitted wave refracted at $45^{\circ}$. (b) When a control wave (in red) is impinging on the metasurface, the transmitted wave refraction angle becomes $-45^{\circ}$. Note that both signal and control waves have the same frequency.

their respective sources would therefore overlap. To overcome this difficulty, we propose the configuration of Figure 10b, where the metasurface presented in Section 3.1.1 is used as a polarization combiner instead of a polarization beam splitter. The physical implementation of the metasurface presented in Figure 10 inherently requires chirality and could therefore not be directly realized with the concept of birefringence. To synthesize the metasurface of Figure 10a, the system (2a) and $(2 \mathrm{~b})$ can be solved by specifying appropriate scattering parameters, as discussed in references [4,6]. One possible set of scattering parameters is given here

$$
\begin{aligned}
& \overline{\overline{S_{21}}}=\frac{\sqrt{2}}{2}\left(\begin{array}{cc}
0 & 0 \\
-1 & 1
\end{array}\right), \\
& \overline{\overline{S_{12}}}=\frac{\sqrt{2}}{2}\left(\begin{array}{cc}
0 & -1 \\
0 & 1
\end{array}\right),
\end{aligned}
$$

where port 1 corresponds to the incident side of Figure 10a (left-hand side) and port 2 corresponds to the transmit side (right-hand side). The reflection scattering tensors $\overline{\overline{S_{11}}}$ and $\overline{\overline{S_{22}}}$ are here left as free parameters. Due to the chiral nature of the metasurface, the structure is bi-anisotropic irrespectively to the values of $\overline{\overline{S_{11}}}$ and $\overline{\overline{S_{22}}}$. An important point, apparent in relations (7a) and (7b), is that the efficiency of the metasurface switch described here is limited to $50 \%$. This can be understood by considering a $y$-polarized normally incident plane wave from port 2. As given by (7b), the $y$-polarized incident wave splits equally into two waves respectively polarized along $x$ and $y$. Since the metasurface is reciprocal (i.e. $\overline{\overline{S_{21}}}={\overline{\overline{S_{12}}}}^{T}$, as given by the Jones matrix calculus [33]), the transmission of either the signal or the control wave is limited to $50 \%$. Higher efficiency could be achieved with a non-reciprocal metasurface which is the topic of the next section.

To implement the metasurface switch, the three metallic layer approach is used. To obtain the chiral specified behavior, the central metallic layer is rotated $45^{\circ}$, as shown in Figure 11 . 


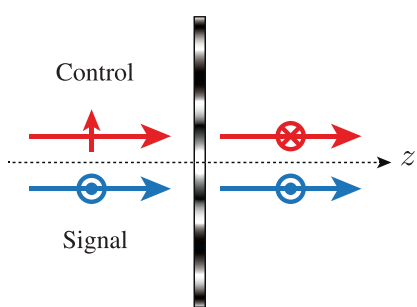

(a)

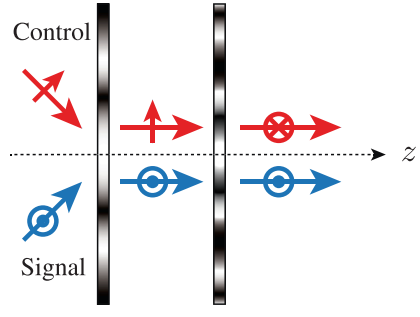

(b)
Figure 10. Representations of the metasurface switch concept. (a) The control and the signal waves are both normally incident on the metasurface. The polarization of the control wave is rotated to match the polarization of the signal wave. A $\pi$-phase shift is imposed between the two transmitted waves, so that they cancel each other by destructive interference. (b) The control and the signal waves are first combined together by the polarization combiner described in Section 3.1.1.

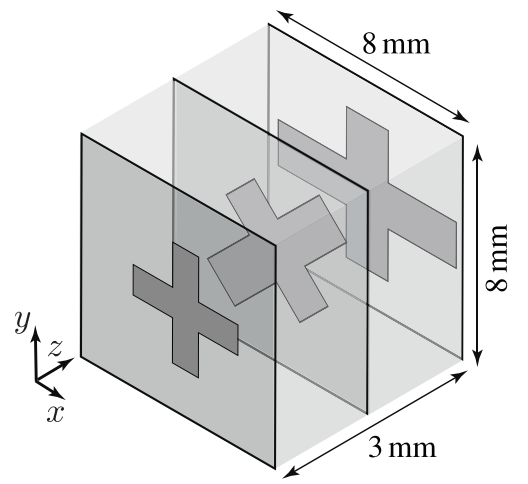

(a)

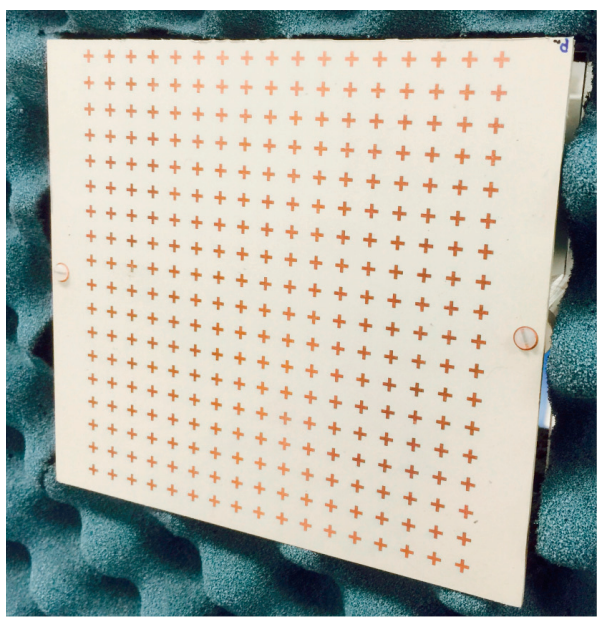

(b)

Figure 11. (a) Representation of a three layer unit cell of the metasurface switch. (b) Fabricated structure, with $17 \times 18$ unit cells, on the measurement stage.

Simulations of the metasurface when illuminated by the signal wave, the control wave and with the two waves simultaneously is shown in Figures 12a-12c, respectively.

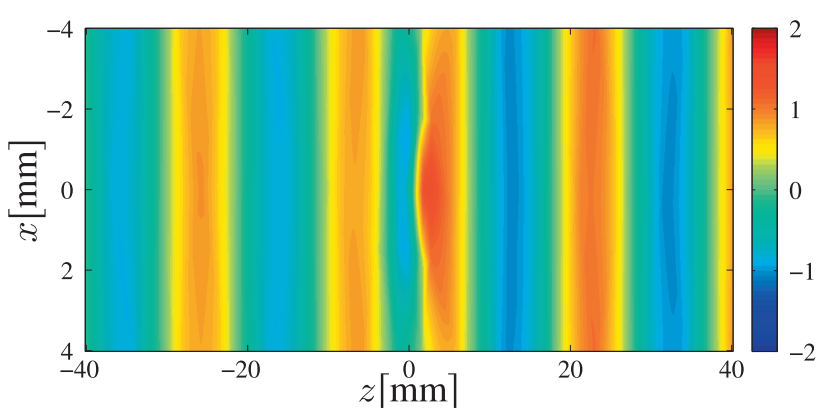

(a)

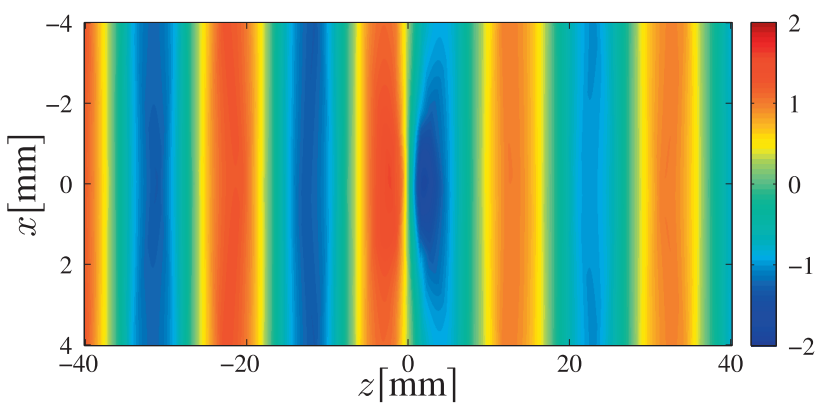

(b)

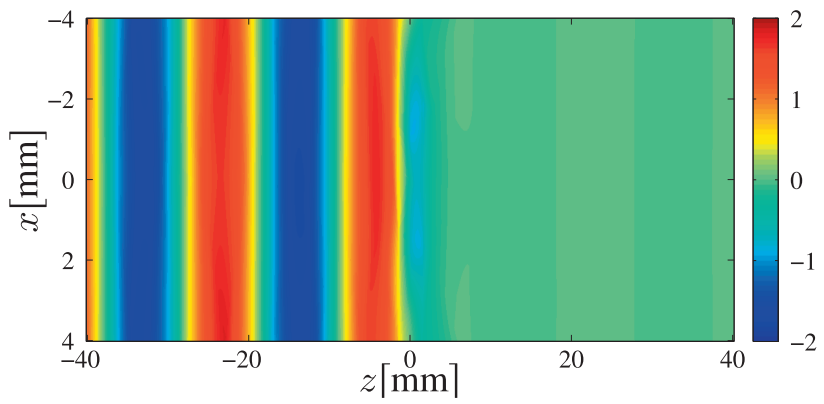

(c)

Figure 12. Numerical simulations (CST) of the $y$-polarized fields, the metasurface is at $z=0$. (a) Fields when only the control wave is incident on the structure. The control wave is initially $x$-polarized (not shown here) from left to right. In the positive $z$-direction, the control wave is transmitted with a $y$ polarization. In the negative $z$-direction, the control wave is reflected with a $y$ polarization. (b) Fields when only the signal wave is incident on the metasurface. (c) Combination of control and signal waves, the cancellation of the transmission by destructive interference is clearly visible.

\subsection{Non-reciprocal non-gyrotropic metasurface}

Non-reciprocal devices are usually based on magnetic material non-reciprocal gyrotropy, whose Faraday rotation is a particular effect $[34,35]$. Recently, non-reciprocal gyrotropic metasurfaces, having a similar response to magnetic materials but without requiring a magnet, have been reported [36-38].

We will discuss here a one-wave transparent metasurface that does not induce polarization rotation and whose principle is shown in Figure 13a. The specified response of the structure is

$$
\overline{\overline{S_{21}}}=\left(\begin{array}{ll}
1 & 0 \\
0 & 1
\end{array}\right)
$$




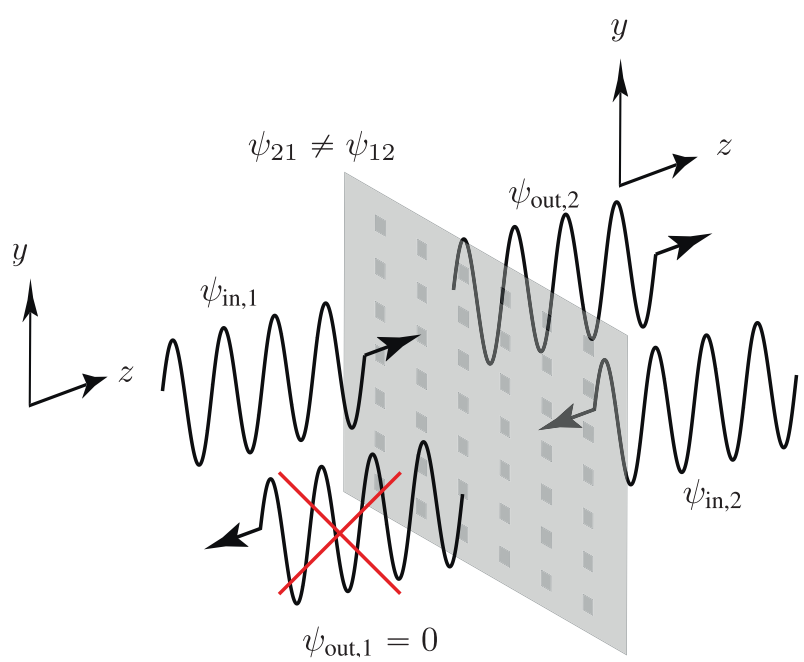

(a)

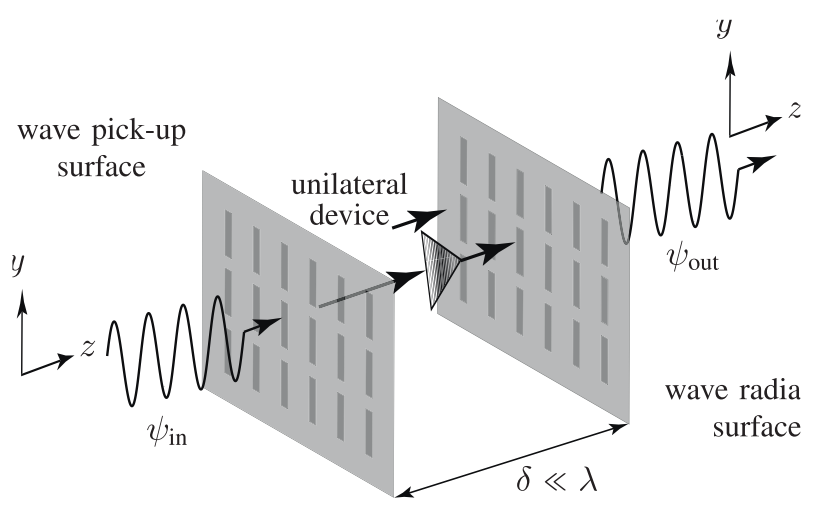

(b)

Figure 13. Spatial nonreciprocal nongyrotropic metasurface. (a) Functional illustration. (b) Proposed pick up-circuit-radiator (PCR) implementation.

while the other scattering parameters are $\overline{\overline{S_{12}}}=\overline{\overline{S_{11}}}=$ $\overline{\overline{S_{22}}}=0$. Solving the system (2a) and (2b), following the procedure described in references $[4,6]$, with the specified scattering parameters, yields the susceptibility tensors

$$
\begin{array}{ll}
\overline{\bar{\chi}}_{\mathrm{ee}}=-\frac{j}{k}\left(\begin{array}{cc}
1 & 0 \\
0 & 1
\end{array}\right), & \overline{\bar{\chi}}_{\mathrm{mm}}=-\frac{j}{k}\left(\begin{array}{cc}
1 & 0 \\
0 & 1
\end{array}\right), \\
\overline{\bar{\chi}}_{\mathrm{em}}=\frac{j}{k}\left(\begin{array}{cc}
0 & 1 \\
-1 & 0
\end{array}\right), & \overline{\bar{\chi}}_{\mathrm{me}}=\frac{j}{k}\left(\begin{array}{cc}
0 & -1 \\
1 & 0
\end{array}\right) .
\end{array}
$$

The non-reciprocal behavior of the metasurface is evident from the fact that the susceptibility tensors $(9 b)$ dot not respect the reciprocity condition, i.e. violate $\overline{\bar{\chi}}_{\mathrm{em}}=-\overline{\bar{\chi}}_{\mathrm{me}}^{T}$. It can be easily understood why the synthesized metasurface needs to be bi-anisotropic even though no rotation of polarization is specified: because it is specified to be non-gyrotropic, the non-diagonal elements of $\overline{\bar{\chi}}_{\mathrm{ee}}$ and $\overline{\bar{\chi}}_{\mathrm{mm}}$, as well as the diagonal elements of $\overline{\bar{\chi}}_{\mathrm{em}}$ and $\overline{\bar{\chi}}_{\mathrm{me}}$, are necessarily zero since they correspond to gyrotropic behavior. Consequently, the tensors $\overline{\bar{\chi}}_{\mathrm{ee}}$ and $\overline{\bar{\chi}}_{\mathrm{mm}}$, being diagonal, can only correspond to reciprocal

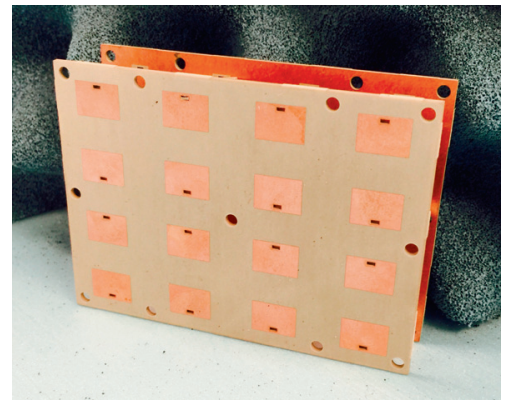

(a)

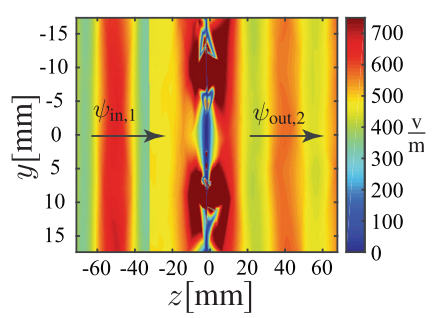

(b)

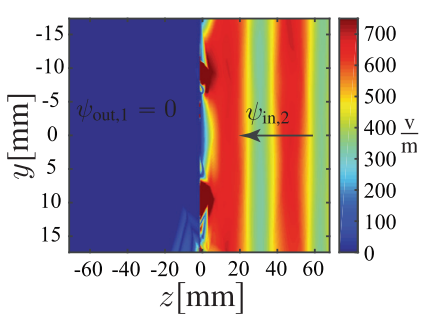

(c)
Figure 14. (a) Fabricated non-reciprocal non-gyrotropic metasurface with 16 unit cells. Full-wave simulated electric fields of the metasurface loaded with a perfect isolator when: (b) excited from port 1 and (c) excited from port 2. The operating frequency is $9.5 \mathrm{GHz}$.

behavior according to the reciprocity conditions (i.e. $\overline{\bar{\chi}}_{\mathrm{ee}}=\overline{\bar{\chi}}_{\mathrm{ee}}^{T}$ and $\overline{\bar{\chi}}_{\mathrm{mm}}=\overline{\bar{\chi}}_{\mathrm{mm}}^{T}$ ). The remaining way to simultaneously achieve non-reciprocity and non-gyrotropy in such case is by leveraging bi-anisotropy. The metasurface described by relations (9a) and (9b) is a particular case of a one-way transparent sheet [39]. The magnetoelectric coupling coefficients, given in (9b), have non-zero asymmetric non-diagonal terms, which classifies this kind of structure as a moving medium [40].

Because the structure described by relations (9a) and (9b) is not trivial to implement with the tools given in Section 2.2, we have used an alternative approach based on a similar design to the one used in reference [41]. The metasurface, as shown in Figure 13b, consists in a pick-up circuit radiator (PCR) structure. The pick-up and radiator faces of the metasurface are composed of patch antennas linked together by an electric circuit present in the center of the structure. When the electric circuit is loaded with an insulating device (like a FET transistor), transmission is allowed only in one direction, effectively realizing the specified scattering parameters. More details about the implementation of this metasurface will be presented elsewhere. Figure 14 shows the simulated field when the metasurface is illuminated from left to right or from right to left, the non-reciprocal behavior can clearly be seen.

The realization of non-reciprocal metasurfaces may become an important part in the implementation of more complicated metamaterial structures allowing more complex field transformations as, for example, the metasurface switch discussed in the previous section. 


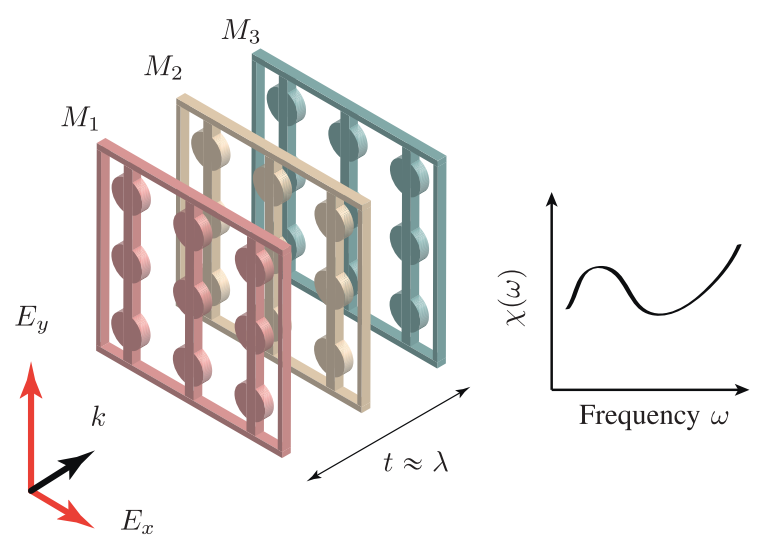

Figure 15. Conceptual idea of cascading several dielectric metasurfaces for dispersion engineering.

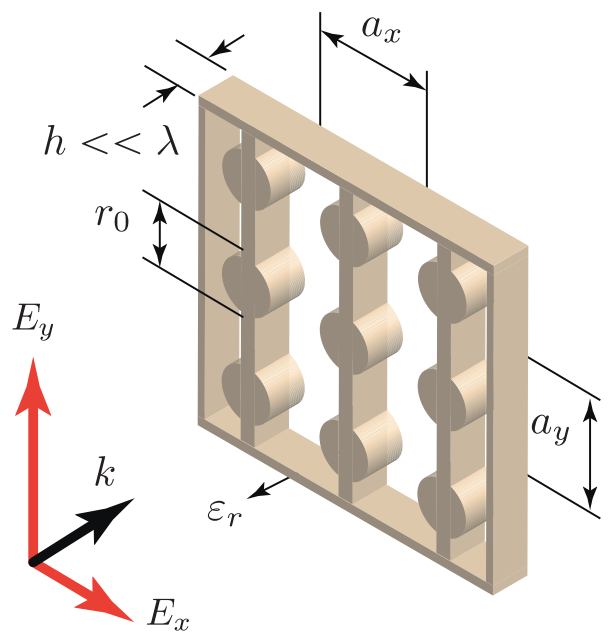

(a)

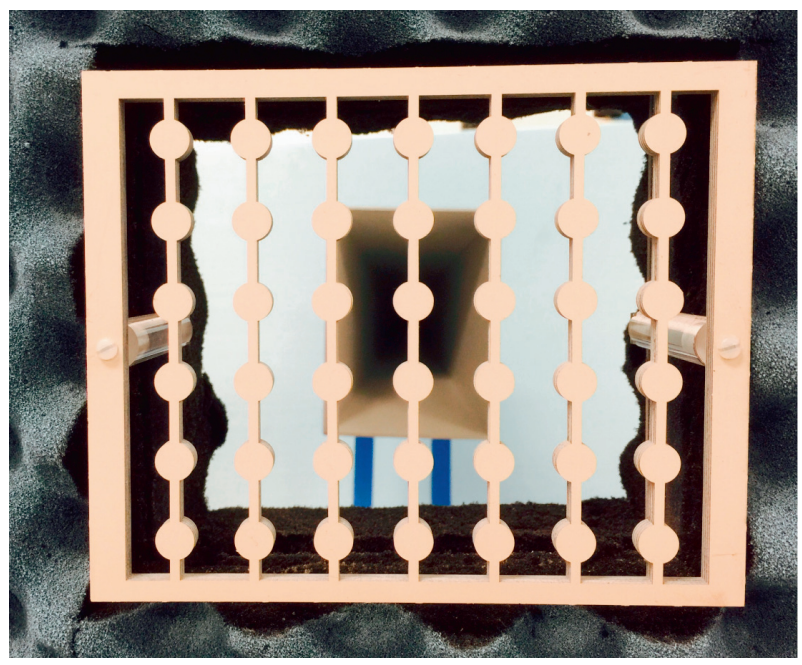

(b)

Figure 16. Proposed all-pass and all-dielectric metasurface based on 2-D array of inter-connected coupled cylinders. (a) Structure illustration. (b) Fabricated prototype, the exciting horn antenna is visible in the background.
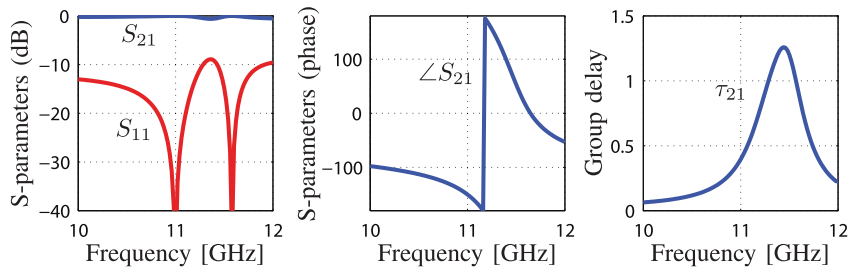

Figure 17. Rigorous coupled wave analysis (RCWA) of the metasurface of Figure 16b. The plots show the transmission and reflection coefficients as well as the transmission phase and the transmission group delay, respectively. The field is polarized in the $x$ direction (perpendicular to the dielectric interconnections).

\subsection{Frequency dispersion engineering}

So far, most existing metasurfaces perform spatial transformations for monochromatic waves only. As metasurface applications will become more and more advanced, the demand for non-uniform phase control over large frequency bands will increase. As it stands, the synthesis technique of Section 2.1 is restricted to monochromatic wave transformations. However, (2a) and (2b) may be solved repeatedly for different frequencies, consequently forming frequency-dependent, or temporally-dispersive, susceptibility components, i.e. $\chi(\omega)$. The response of the metasurface could therefore be engineered for the specified frequency band in the similar fashion that phasers can achieve real-time analog signal processing (R-ASP) [42].

One possible approach, to engineer frequency-dependent metasurfaces is to use the additional degrees of freedom provided by coupled resonators as demonstrated in reference [43]. Another possibility, that is proposed here, is to cascade metasurfaces made of dielectric scattering particles, as depicted in Figure 15. As shown in Section 2.2.2, cylindrical dielectric resonators can be tuned to achieve near full transmission and $2 \pi$ phase coverage over a specific bandwidth. The proposed all-pass metasurface is shown in Figure 16. In this configuration, each cylinder is mechanically supported by dielectric interconnections, which also suppress spurious Fabry-Pérot resonances that would occur if the dielectric particles were placed on a supporting slab.

The simulated response of the dielectric metasurface of Figure 16b is presented in Figure 17. A flat and almost full transmission is achieved over a large bandwidth while a full phase cycle occurs around the resonant frequency $\omega=11.15 \mathrm{GHz}$.

\section{Conclusion}

In this paper, we have presented a partial overview of the synthesis and possible applications of electromagnetic metasurfaces and several illustrative examples of metasurface applications, in an increasing order of synthesis complexity. First, the concept of birefringence, which allows an independent control of both orthogonal polarizations, was used to implement two diffraction engineering metasurfaces: one for polarization dependent generalized refraction and the other one for orbital angular momentum multiplexing. Next, the implementation 
of an electromagnetic remotely controlled metasurface spatial processor was discussed. It turns out that birefringence, which is based on anisotropy, is fundamentally insufficient to realize this remotely controlled metasurface processor, the latter requiring bi-anisotropic constituents. It was shown that the efficiency of such metasurfaces is limited due to its inherent reciprocal operating principle. However, a non-reciprocal implementation of these metasurfaces could theoretically achieve a much better efficiency. Based on this consideration, a non-reciprocal non-gyrotropic metasurface was introduced. This metasurface, which also requires bi-anisotropic features, could be a possible future solution to realize efficient nonreciprocal remotely controlled metasurface processors. Finally, we have introduced a conceptual idea to realize all-pass dispersion engineering metasurfaces able to control the group delay of electromagnetic signals of large bandwidth, which constitute a missing piece in the metasurface arsenal.

\section{References}

1. C. Holloway, A. Dienstfrey, E.F. Kuester, J.F. O'Hara, A.K. Azad, A.J. Taylor, A discussion on the interpretation and characterization of metafilms/metasurfaces: the two-dimensional equivalent of metamaterials, Metamaterials 3 (2009) 100-112.

2. C. Holloway, E.F. Kuester, J. Gordon, J. O'Hara, J. Booth, D. Smith, An overview of the theory and applications of metasurfaces: the two-dimensional equivalents of metamaterials, IEEE Antennas Propag. Mag. 54 (2012) 10-35.

3. N. Yu, F. Capasso, Flat optics with designer metasurfaces, Nature Mater. 13 (2014) 139-150.

4. K. Achouri, M.A. Salem, C. Caloz, General metasurface synthesis based on susceptibility tensors, IEEE Trans. Antennas Propag. 63 (2015) 2977-2991.

5. M.A. Salem, C. Caloz, Manipulating light at distance by a metasurface using momentum transformation, Opt. Express 22 (2014) 14530-14543.

6. C. Pfeiffer, A. Grbic, Bianisotropic metasurfaces for optimal polarization control: Analysis and synthesis, Phys. Rev. Appl. 2 (2014) 044011.

7. T. Niemi, A. Karilainen, S. Tretyakov, Synthesis of polarization transformers, IEEE Trans. Antennas Propag. 61 (2013) 3102-3111.

8. M. Selvanayagam, G. Eleftheriades, Polarization control using tensor huygens surfaces, IEEE Trans. Antennas Propag. 62 (2014) 6155-6168.

9. M.M. Idemen, Discontinuities in the electromagnetic field, John Wiley \& Sons, New York, 2011.

10. E.F. Kuester, M. Mohamed, M. Piket-May, C. Holloway, Averaged transition conditions for electromagnetic fields at a metafilm, IEEE Trans. Antennas Propag. 51 (2003) 2641-2651.

11. A.H. Sihvola, A.J. Viitanen, I.V. Lindell, S.A. Tretyakov, Electromagnetic waves in chiral and bi-isotropic media, ser. The Artech House Antenna Library, Artech House, Norwood, Massachusetts, 1994.

12. B.A. Munk, Frequency selective surfaces: theory and design, John Wiley \& Sons, New York, 2000.

13. F. Monticone, N.M. Estakhri, A. Alù, Full control of nanoscale optical transmission with a composite metascreen, Phys. Rev. Lett. 110 (2013) 203903.
14. C. Pfeiffer, A. Grbic, Millimeter-wave transmitarrays for wavefront and polarization control, IEEE Trans. Microwave Theory Tech. 61 (2013) 4407-4417.

15. J.-S.G. Hong, M.J. Lancaster, Microstrip filters for RF/microwave applications, Vol. 167, John Wiley \& Sons, New York, 2004.

16. G. Mie, Beiträge zur optik trüber medien, speziell kolloidaler metall ösungen, Annalen der Physik 330 (1908) 377-445.

17. M. Kerker, The scattering of light and other electromagnetic radiation: physical chemistry: a series of monographs, Vol. 16, Academic press, Cambridge, Massachusetts, 2013.

18. A. Krasnok, S. Makarov, M. Petrov, R. Savelev, P. Belov, Y. Kivshar, Towards all-dielectric metamaterials and nanophotonics, Proc. SPIE 9502 (2015) 950203-950217.

19. A. Arbabi, Y. Horie, M. Bagheri, A. Faraon, Dielectric metasurfaces for complete control of phase and polarization with subwavelength spatial resolution and high transmission, Nature Nanotech. 10 (2015) 937-943.

20. V.S. Asadchy, I.A. Faniayeu, Y. Ra'di, S.A. Khakhomov, I.V. Semchenko, S.A. Tretyakov, Broadband reflectionless metasheets: frequency-selective transmission and perfect absorption, Phys. Rev. X 5 (2015) 031005.

21. B. Saleh, M. Teich, Fundamentals of Photonics, ser. Wiley Series in Pure and Applied Optics, Wiley, New York, 2007.

22. J. Nye, M. Berry, Dislocations in wave trains, Philos. Trans. R. Soc. Lond. Ser. B: Math. Phys. Eng. Sci. 336 (1974) 165-190.

23. E. Karimi, S.A. Schulz, I. De Leon, H. Qassim, J. Upham, R.W. Boyd, Generating optical orbital angular momentum at visible wavelengths using a plasmonic metasurface, Light: Science \& Applications 3 (2014) e167.

24. X. Yi, X. Ling, Z. Zhang, Y. Li, X. Zhou, Y. Liu, S. Chen, H. Luo, S. Wen, Generation of cylindrical vector vortex beams by two cascaded metasurfaces, Opt. Express 22 (2014) 17207-17215.

25. C. Pfeiffer, A. Grbic, Controlling vector bessel beams with metasurfaces, Phys. Rev. Appl. 2 (2014) 044012.

26. E. Karimi, G. Zito, B. Piccirillo, L. Marrucci, E. Santamato, Hypergeometric-gaussian beams, Opt. Lett. 32 (2007) 3053-3055.

27. S.N. Burokur, J.-P. Daniel, P. Ratajczak, A. de Lustrac, Tunable bilayered metasurface for frequency reconfigurable directive emissions, Appl. Phys. Lett. 97 (2010) 064101.

28. A. Fallahi, J. Perruisseau-Carrier, Design of tunable biperiodic graphene metasurfaces, Phys. Rev. B 86 (2012) 195408.

29. S. Saadat, H. Mosallaei, Tunable and active metasurface-based onchip antennas, Antennas and Propagation Society International Symposium (APSURSI), 2012, IEEE, Chicago, 2012, pp. 1-2.

30. B.O. Zhu, J. Zhao, Y. Feng, Active impedance metasurface with full $360^{\circ}$ reflection phase tuning, Sci. Rep. 3 (2013) 3059.

31. Z. Xie, X. Wang, J. Ye, S. Feng, W. Sun, T. Akalin, Y. Zhang, Spatial terahertz modulator, Sci. Rep. 3 (2013) 3347.

32. S.E. Harris, Electromagnetically induced transparency, Phys. Today 50 (2008) 36-42.

33. R.C. Jones, A new calculus for the treatment of optical systems. I. Description and discussion of the calculus, J. Opt. Soc. Am. 31 (1941) 488-493.

34. C.L. Hogan, The ferromagnetic Faraday effect at microwave frequencies and its applications, Bell Syst. Tech. J. 31 (1952) 1-31.

35. C. Hogan, The ferromagnetic Faraday effect at microwave frequencies and its applications, Rev. Mod. Phys. 25 (1953) 253. 
36. T. Kodera, C. Caloz, Non-reciprocal magnetic frequency selective surface Antennas and Propagation, 2009. EuCAP 2009. 3rd European Conference on, IEEE, Berlin, 2009, pp. $1552-1555$.

37. T. Kodera, A. Parsa, C. Caloz, Non-reciprocal ferrite antenna radome: the faradome, Antennas and Propagation Society International Symposium, 2009. APSURSI'09, IEEE, North Charleston, SC, USA, 2009, pp. 1-4.

38. A. Parsa, T. Kodera, C. Caloz, Ferrite based non-reciprocal radome, generalized scattering matrix analysis and experimental demonstration, IEEE Trans. Antennas Propag. 59 (2011) 810-817.

39. Y. Ra'di, V.S. Asadchy, S.A. Tretyakov, One-way transparent sheets, Phys. Rev. B 89 (2014) 075109.
40. S.A. Tretyakov, A. Sihvola, A. Sochava, C. Simovski, Magnetoelectric interactions in bi-anisotropic media, J. Electromagn. Waves Appl. 12 (1998) 481-497.

41. Z. Wang, Z. Wang, J. Wang, B. Zhang, J. Huangfu, J.D. Joannopoulos, M. Soljačić, L. Ran, Gyrotropic response in the absence of a bias field, Proc. Natl. Acad. Sci. 109 (2012) 13194-13197.

42. C. Caloz, S. Gupta, Q. Zhang, B. Nikfal, Analog signal processing, Microw. Mag. 14 (2013) 87-103.

43. F. Aieta, M.A. Kats, P. Genevet, F. Capasso, Multiwavelength achromatic metasurfaces by dispersive phase compensation, Science 347 (2015) 1342-1345.

Cite this article as: Achouri K, Khan BA, Gupta S, Lavigne G, Salem MA \& Caloz C: Synthesis of electromagnetic metasurfaces: principles and illustrations. EPJ Appl. Metamat. 2015, 2, 12. 